\title{
BMJ Open Incidence of major complications after percutaneous native renal biopsies in adults from low-income to middle- income countries: a protocol for systematic review and meta-analysis
}

\author{
Shepherd Kajawo, ${ }^{1,2,3}$ Mothusi Walter Moloi, ${ }^{1,3}$ Jean Jacques Noubiap, ${ }^{4}$ \\ Udeme Ekrikpo, ${ }^{1,5}$ Andre Pascal Kengne, ${ }^{4,6}$ Ikechi G Okpechi ${ }^{1,3}$
}

To cite: Kajawo S, Moloi MW, Noubiap JJ, et al. Incidence of major complications after percutaneous native renal biopsies in adults from lowincome to middle-income countries: a protocol for systematic review and meta-analysis. BMJ Open 2018;8:e020891. doi:10.1136/ bmjopen-2017-020891

- Prepublication history for this paper is available online. To view these files, please visit the journal online (http://dx.doi. org/10.1136/bmjopen-2017020891).

Received 30 November 2017 Revised 13 February 2018 Accepted 7 March 2018

Check for updates

For numbered affiliations see end of article.

Correspondence to

Dr. Shepherd Kajawo; skajawo@gmail.com

\section{ABSTRACT}

Introduction Kidney biopsy is an essential tool for guiding clinicians towards diagnoses, treatment and determining prognosis in renal disease. However, the procedure can be marred by various complications. The reported occurrence of complications varies among countries or regions and is also affected by several clinical and technical factors. This systematic review and meta-analysis aims to evaluate the incidence of major complications after percutaneous native renal biopsy in low-income to middle-income countries (LMICS).

Methods and analysis We will include studies of populations from LMIC as per World Bank 2017 country list. Relevant abstracts published from 1 January 1980 to 30 December 2017 will be searched in PubMed, Cochrane, Excerpta Medica Database (Embase) and African Journals Online, without language restriction. Two reviewers will independently screen, select studies, extract data and assess the risk of bias in each study. A third reviewer will arbitrate in cases of disagreements. The study-specific estimates will be pooled through a random-effects model meta-analysis to obtain an overall summary estimate of the incidence of major complications across studies. Clinical and statistical heterogeneity will be evaluated by Cochrane's $Q$ statistic. Funnel-plot analysis and Egger's test will be used to assess publication bias. Results will be presented by geographical region and income group. Ethics and dissemination This study will use published data. Therefore, there is no requirement for ethical approval. This systematic review and meta-analysis is expected to inform healthcare workers and providers about the occurrence of major complications following renal biopsies and highlight possible actions needed to improve the safety of the procedure in LMICs. The final report will be published as an original article in a peer-reviewed journal. Findings will also be presented at a conference and submitted to relevant health authorities.

PROSPERO registration number CRD42017077656.

\section{INTRODUCTION}

Kidney biopsy is an essential tool for guiding clinicians towards the diagnosis of unexplained renal disease, guide treatment of

\section{Strengths and limitations of this study}

- To our knowledge, this will be the first systematic review and meta-analysis aiming to estimate the incidence of complications after renal biopsies in low-income to middle-income countries.

- We aim to look at the distribution of biopsy complications according to income group, providing comparisons that may be useful in designing strategies to improve safety of renal biopsies in this region.

- There could be heterogeneity from studies from different regions, which may affect pooled estimates.

- The limited number of patients having renal biopsies in some countries may affect true incident rates.

patients or assist with determination of prognosis of renal disease. ${ }^{1}$ Since its first introduction in 1944 and later in 1951 by Iversen and Brun, the procedure has provided clinicians with valuable information about kidney diseases and their management despite high rates of reported biopsy complications. ${ }^{2}$ Improvements of the technique and recent introduction of the spring-loaded biopsy gun have reduced some of these complications. ${ }^{2}$

The reported occurrence of complications following a kidney biopsy tend to vary from one region to another. A study from China, a middle-income country, reported incidence of severe bleeding to be $0.39 \%$ out of 3577 native kidney biopsies. ${ }^{3}$ However, much higher rates have been reported in other parts of the world. ${ }^{4-6}$ In Morocco, $12.3 \%$ of patients who had undergone a percutaneous native kidney biopsy had gross haematuria and $0.8 \%$ required nephrectomy after biopsy. ${ }^{4}$ On the other hand, a systematic review with studies mainly from high-income countries found that only $0.9 \%$ of 9474 renal biopsies required red blood cell transfusion. ${ }^{7}$ 
However, the review only included studies if renal biopsy was performed by real-time ultrasound using automatic biopsy needles. ${ }^{7}$ Due to lack of such equipment in developing countries, no low-income to middle-income country (LMIC) was included in that study, making their results difficult to generalise globally.

The use of automated spring-loaded biopsy devices under real-time ultrasound guidance for performance of renal biopsy is becoming increasingly common in many centres. ${ }^{23}$ However, there are studies that have shown that the spring-driven needles may yield similar complication rates when compared with hand-driven needles. ${ }^{8}$ Similarly, while minor complications have been shown to be increased with use of free hand rather than realtime ultrasound, the incidence of major complications has been shown to be similar in both techniques. ${ }^{9}$ This is further supported by studies reporting no difference in diagnostic yield or occurrence of complications between ultrasound marked or blind biopsies. ${ }^{8} 10$ Other factors that may influence the rates of complications are the size of needle used as some studies show that large-calibre needles such as the $14 \mathrm{G}$ needle may result in higher bleeding complications when compared with smaller needles. ${ }^{7}$ Other studies have, however, found no difference in complication rates based on size of needle used for the procedure. ${ }^{211}$ Operator experience and training may also affect the reported incidence of renal biopsy complications. ${ }^{10}$ Finally, despite the increasing number of kidney biopsies being performed by radiologists, there is no evidence that they are safer than those performed by nephrologists. ${ }^{11} 12$

This systematic review aims to summarise available data on the incidence of reported complications in patients undergoing percutaneous renal biopsy in LMICs. It is anticipated this will provide essential data to aid the global strategy in curbing kidney disease in LMIC. ${ }^{13}$ This is important as it will provide information regarding the safety of performing renal biopsies, especially in LMICs where glomerular diseases are common causes of chronic kidney disease and remain largely undiagnosed. ${ }^{14}$

\section{RESEARCH OBJECTIVES}

This review aims to report on the incidence of major complications associated with performance of renal biopsies in LMIC.

\section{REVIEW QUESTION}

What is the incidence of major complications after renal biopsy in adults undergoing percutaneous renal biopsy in LMIC?

We will define major complications as any complication requiring an intervention, for example blood transfusion, radiographic or surgical invasive procedure, acute renal obstruction, extended hospitalisation, septicaemia or death. Minor complications will be those that are expected to resolve spontaneously, for example, pain after biopsy or mild haematuria resolving spontaneously without the need for blood transfusion or other interventions.

\section{METHODS}

The development of this protocol was done using the Preferred Reporting Items for Systematic reviews and Meta-Analyses for Protocols 2015 (PRISMA-P 2015) framework. $^{15}$

\section{Criteria for considering studies for the review \\ Inclusion criteria}

1. Studies done in LMICs reporting on complications after percutaneous native renal biopsies from 1 January 1980 to 30 December 2017 will be included. We will focus on the period after 1980 given that the techniques of renal biopsies changed substantially after 1980 with increased use of ultrasound and automatic needles. ${ }^{216}$

2. We will consider relevant cohort, cross-sectional or case-control studies from LMIC.

3. Studies with patients 18 years and above.

\section{Exclusion criteria}

1. Studies reporting biopsies of tumours or kidney masses.

2. Studies with fewer than 30 patients.

3. Editorials and review articles.

4. Studies from high-income countries.

5. Studies reporting on kidney transplant biopsies only.

6. Studies with duplicate publications. In such cases, we will consider only the most up-to-date and comprehensive publication.

7. Studies with inaccessible data even after request from the authors.

\section{Search strategy for identifying relevant studies}

1. Bibliographic database searches: We will search PubMed, Excerpta Medica Database (Embase), Cochrane and African Journals Online databases for relevant abstracts. The search will be performed from 1 January 1980 to 30 December 2017 with no language restriction (table 1). Conference abstracts and grey literature studies will also be considered. Dissertations and theses will be searched from ProQuest Dissertations, Theses Online, Networked Digital Library of Theses and Dissertations and the Open Access.

2. All relevant abstracts from above sources will be scrutinised and full papers downloaded from the databases or journal websites. The references of these papers will be checked for potentially eligible studies, which will be assessed for eligibility.

Selection of studies deemed relevant for inclusion in the review Two reviewers (SK and MWM) will independently search the databases for relevant abstracts using agreed study inclusion and exclusion criteria. The full texts of these abstracts will again be independently assessed by these 
Table 1 Search strategy for PubMed

\#1 Kidney biopsy OR Renal biopsy

\#2 Complications OR Adverse events OR Adverse effects OR haematoma OR Hematoma OR hemorrhage OR haemorrhage OR bleeding OR blood transfusion OR Red blood cell transfusion OR Erythrocyte transfusion OR red cell transfusion OR cauterisation OR embolisation OR embolization OR Bladder Obstruction OR Arteriovenous fistula OR Arteriovenous aneurysm OR pseudo aneurysm OR Infection OR Angiography OR Needle OR Fine needle OR Ultrasound guided OR nephrectomy OR death

\#3 ((Afghanistan [tiab] OR Albania [tiab] OR Algeria [tiab] OR American Samoa [tiab] OR Angola [tiab] OR Argentina [tiab] OR Armenia [tiab] OR Azerbaijan [tiab] OR Bangladesh [tiab] OR Belarus [tiab] OR Belize [tiab] OR Benin [tiab] OR Bhutan [tiab] OR Bolivia [tiab] OR Bosnia and Herzegovina [tiab] OR Botswana [tiab] OR Brazil [tiab] OR Bulgaria [tiab] OR Burkina Faso [tiab] OR Burundi [tiab] OR Cabo Verde [tiab] OR Cambodia [tiab] OR Cameroon [tiab] OR Central African Republic [tiab] OR Chad [tiab] OR China [tiab] OR Colombia [tiab] OR Comoros [tiab] OR Congo, Dem. Rep. [tiab] OR Congo, Rep. [tiab] OR Costa Rica [tiab] OR Ivory Coast [tiab] OR Cote d'Ivoire [tiab] OR Cuba [tiab] OR Djibouti [tiab] OR Dominica [tiab] OR Dominican Republic [tiab] OR Ecuador [tiab] OR Egypt [tiab] OR El Salvador [tiab] OR Equatorial Guinea [tiab] OR Eritrea [tiab] OR Ethiopia [tiab] OR Fiji [tiab] OR Gabon [tiab] OR Gambia [tiab] OR Georgia [tiab] OR Ghana [tiab] OR Grenada [tiab] OR Guatemala [tiab] OR Guinea [tiab] OR Guinea-Bissau [tiab] OR Guyana [tiab]) OR Haiti [tiab] OR Honduras [tiab] OR India [tiab] OR Indonesia [tiab] OR Iran, Islamic Rep. [tiab] OR Iraq [tiab] OR Jamaica [tiab] OR Jordan [tiab] OR Kazakhstan [tiab] OR Kenya [tiab] OR Kiribati [tiab] OR Korea, Dem. People's Rep. [tiab] OR Kosovo [tiab] OR Kyrgyz Republic [tiab] OR Lao PDR [tiab] OR Lebanon [tiab] OR Lesotho [tiab] OR Liberia [tiab] OR Libya [tiab] OR Macedonia [tiab] OR Madagascar [tiab] OR Malawi [tiab] OR Malaysia [tiab] OR Maldives [tiab] OR Mali [tiab] OR Marshall Islands [tiab] OR Mauritania [tiab] OR Mauritius [tiab] OR Mexico [tiab] OR Micronesia [tiab] OR Moldova [tiab] OR Mongolia [tiab] OR Montenegro [tiab] OR Morocco [tiab] OR Mozambique [tiab] OR Myanmar [tiab] OR Namibia [tiab] OR Nepal [tiab] OR Nicaragua [tiab] OR Niger [tiab] OR Nigeria [tiab] OR Pakistan [tiab] OR Palau [tiab] OR Panama [tiab] OR Papua New Guinea [tiab] OR Paraguay [tiab] OR Peru [tiab] OR Philippines [tiab] OR Romania [tiab] OR Russian Federation [tiab] OR Rwanda [tiab] OR Samoa [tiab] OR São Tomé and Princip [tiab] OR Senegal [tiab] OR Serbia [tiab] OR Sierra Leone [tiab] OR Solomon Islands [tiab] OR Somalia [tiab] OR South Africa [tiab] OR South Sudan [tiab] OR Sri Lanka [tiab] OR St. Lucia [tiab] OR St. Vincent and the Grenadines [tiab] OR Sudan [tiab] OR Suriname [tiab] OR Swaziland [tiab] OR Syrian Arab Republic [tiab] OR Tajikistan [tiab] OR Tanzania [tiab] OR Thailand [tiab] OR Timor-Leste [tiab] OR Togo [tiab] OR Tonga [tiab] OR Tunisia [tiab] OR Turkey [tiab] OR Turkmenistan [tiab] OR Tuvalu [tiab] OR Uganda [tiab] OR Ukraine [tiab] OR Uzbekistan [tiab] OR Vanuatu [tiab] OR Venezuela [tiab] OR Vietnam [tiab] OR West Bank and Gaza [tiab] OR Yemen, Rep. [tiab] OR Zambia [tiab] OR Zimbabwe [tiab]))

\#4 \#1+ \#2 + \#3

\#5 Limits \#4: Humans, 1980/01/01 to 2017/12/30

reviewers for eligibility. Any differences will be resolved by a third reviewer (IGO).

\section{Assessment of methodological quality and reporting of data}

For risk of bias assessment, we will adapt and use a tool developed by Hoy et al to assess the methodological quality of included studies. ${ }^{17}$ This will be presented in a table format.

\section{Data extraction and management}

The data will be extracted onto a predeveloped data capture sheet. We will collect the following information: country of biopsy (in cases of multinational studies, information from individual countries will be presented separately where possible), World Bank Income Group, author, year of publication, study design, sample size, mean age, gender, the reported incidence of various postrenal biopsy complications, method of biopsy (free hand, ultrasound guided or ultrasound marking), needle size, type of needle used for the biopsy (automatic, manual), indication of biopsy, factors associated with major bleeding, for example, elevated blood pressure, platelet count and coagulation parameters, and operator (nephrologist, radiologist, trainee).

\section{Statistical analysis}

A meta-analysis will be used to summarise incidence data. We will pool the study-specific estimates using a random-effects meta-analysis model (DerSimonian-Laird) to obtain an overall summary estimate of the incidence of complications across studies. ${ }^{18}$ The incidence rates follow a Poisson distribution. Therefore, the square-root transformation will be applied to stabilise the variance of the incidence rates prior to meta-analyses, and the estimates back-transformed for reporting. Heterogeneity will be assessed using the $\chi^{2}$ test on Cochrane's $Q$ statistic ${ }^{19}$ and quantified by calculating the $\mathrm{I}^{2}$ (with values of $25 \%, 50 \%$ and $75 \%$ representing low, medium and high heterogeneity, respectively). ${ }^{20}$ Subgroup analysis will be performed using the Q-test based on ANOVA. We will assess the presence of publication bias using funnel plots and Egger's test. ${ }^{21}$ We will also assess inter-rater agreement for study inclusion and data extraction using Cohen's kappa ( $\kappa)$ coefficient. ${ }^{22} \mathrm{~A}$ $\mathrm{P}$ value $<0.05$ will be considered indicative of statistically significant difference between subgroups. All analyses will be performed using Stata V.15 (StataCorp, College Station, Texas, USA). 


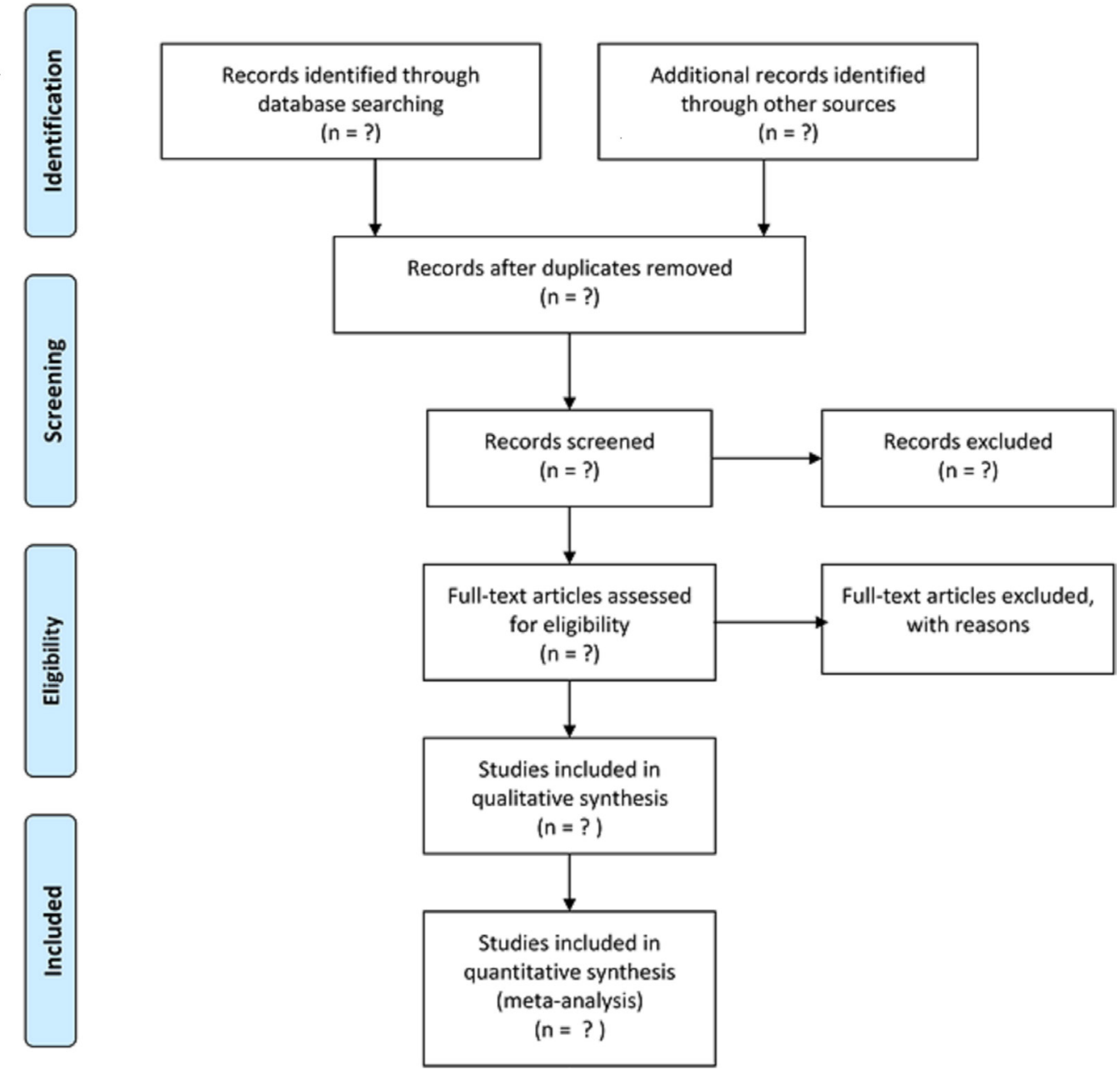

Figure 1 Flow diagram for study selection.

\section{Analysis of subgroups or subsets}

The following subgroups will be considered for analysis:

1. Gender: male versus female.

2. Geographic location, income group (low income versus middle income) as per 2017 World Bank country list.

3. Needle size ( $14 \mathrm{G}$ vs $16 \mathrm{G}$ vs $18 \mathrm{G}$ ).

4. Biopsy technique (blind vs real time vs ultrasound assisted).

5. Needle type (spring loaded versus manual).

\section{Reporting of results and presentation}

A PRISMA flow diagram will be used to present the study selection process (figure 1). We will use the PRISMA Guidelines to present our data. ${ }^{23}$ Quantitative data will be presented in tables and forest plots if applicable. The prevalent complication rates in various LMIC regions will be presented and comparisons made.

\section{CONCLUSION}

Kidney biopsies are essential to help manage kidney disease. Unfortunately, access to this resource is limited in poorly resourced countries. It is important to gather accurate data on renal biopsies from LMIC to aid policy-making and improvements to be made where necessary. We hope this review will help to highlight challenges faced in performing renal biopsies in this region.

\section{Author affiliations}

${ }^{1}$ Division of Nephrology and Hypertension, University of Cape Town, Cape Town, Western Cape, South Africa

${ }^{2}$ Division of Clinical Practice and Patient Care, National University of Science and Technology, Bulawayo, Zimbabwe

${ }^{3}$ Kidney and Hypertension Research Unit, University of Cape Town, Cape Town, South Africa

${ }^{4}$ Department of Medicine, Groote Schuur Hospital and University of Cape Town, Cape Town, South Africa

${ }^{5}$ Renal Unit, Department of Medicine, University of Uyo, Uyo, Nigeria ${ }^{6}$ Non-Communicable Diseases Research Unit, Medical Research Council of South Africa, Cape Town, South Africa

Contributors SK, JJN and IGO conceived and designed the protocol of this study. SK drafted the manuscript. SK and MWM will do the search and IGO will arbitrate any differences. UE and APK helped in the revision of the manuscript and methodological design of the study. UE and APK will help with the statistics and the meta-analysis. IGO is the guarantor of the review. All authors approved the final version of this protocol.

Funding The authors have not declared a specific grant for this research from any funding agency in the public, commercial or not-for-profit sectors.

Competing interests None declared.

Patient consent Not required. 
Provenance and peer review Not commissioned; externally peer reviewed.

Open Access This is an Open Access article distributed in accordance with the Creative Commons Attribution Non Commercial (CC BY-NC 4.0) license, which permits others to distribute, remix, adapt, build upon this work non-commercially, and license their derivative works on different terms, provided the original work is properly cited and the use is non-commercial. See: http://creativecommons.org/ licenses/by-nc/4.0/

(C) Article author(s) (or their employer(s) unless otherwise stated in the text of the article) 2018. All rights reserved. No commercial use is permitted unless otherwise expressly granted.

\section{REFERENCES}

1. Fiorentino M, Bolignano D, Tesar V, et al. Renal biopsy in 2015from epidemiology to evidence-based indications. Am J Nephrol 2016;43:1-19.

2. Visconti L, Cernaro V, Ricciardi CA, et al. Renal biopsy: still a landmark for the nephrologist. World J Nephrol 2016;5:321-7.

3. Xu DM, Chen M, Zhou FD, et al. Risk factors for severe bleeding complications in percutaneous renal biopsy. Am J Med Sci 2017;353:230-5

4. Zajjari Y, Aatif T, Bahadi A, et al. Kidney biopsy in the Military Hospital of Morocco: complications and histopathological findings. Saudi J Kidney Dis Transpl 2015;26:1044-9.

5. Chunduri S, Whittier WL, Korbet SM. Adequacy and complication rates with 14- vs. 16-gauge automated needles in percutaneous renal biopsy of native kidneys. Semin Dial 2015;28:E11-E14.

6. Sobh M, Moustafa F, Ghoniem M. Value of renal biopsy in chronic renal failure. Int Urol Nephrol 1988;20:77-83.

7. Corapi KM, Chen JL, Balk EM, et al. Bleeding complications of native kidney biopsy: a systematic review and meta-analysis. Am J Kidney Dis 2012;60:62-73.

8. Doyle AJ, Gregory MC, Terreros DA. Percutaneous native renal biopsy: comparison of a 1.2-mm spring-driven system with a traditional 2-mm hand-driven system. Am J Kidney Dis 1994;23:498-503.

9. Ali H, Murtaza A, Anderton J, et al. Post renal biopsy complication rate and diagnostic yield comparing hands free (ultrasound-assisted) and ultrasound-guided biopsy techniques of renal allografts and native kidneys. Springerplus 2015;4:491.

10. Hogan JJ, Mocanu M, Berns JS. The native kidney biopsy: update and evidence for best practice. Clin J Am Soc Nephrol 2016;11:354-62.

11. Tøndel C, Vikse BE, Bostad L, et al. Safety and complications of percutaneous kidney biopsies in 715 children and 8573 adults in Norway 1988-2010. Clin J Am Soc Nephrol 2012;7:1591-7.

12. Whittier WL, Korbet SM. Who should perform the percutaneous renal biopsy: a nephrologist or radiologist? Semin Dial 2014;27:243-5

13. Mehta RL, Burdmann EA, Cerdá J, et al. Recognition and management of acute kidney injury in the International Society of Nephrology 0by25 Global Snapshot: a multinational cross-sectional study. Lancet 2016;387:2017-25.

14. Okpechi IG, Ameh OI, Bello AK, et al. Epidemiology of histologically proven glomerulonephritis in Africa: a systematic review and metaanalysis. PLoS One 2016;11:e0152203.

15. Moher D, Shamseer L, Clarke M, et al. Preferred reporting items for systematic review and meta-analysis protocols (PRISMA-P) 2015 statement. Syst Rev 2015;4:1.

16. Wiseman DA, Hawkins R, Numerow LM, et al. Percutaneous renal biopsy utilizing real time, ultrasonic guidance and a semiautomated biopsy device. Kidney Int 1990;38:347-9.

17. Hoy D, Brooks $P$, Woolf $A$, et al. Assessing risk of bias in prevalence studies: modification of an existing tool and evidence of interrater agreement. J Clin Epidemiol 2012;65:934-9.

18. Higgins J, Thompson SG, Deeks JJ, et al. Measuring inconsistency in meta-analyses [journal article as teaching resource, deposited by John Flynn]. British Medical Journal 2003;327:557-60.

19. Cochran WG. The combination of estimates from different experiments. Biometrics 1954;10:101-29.

20. Higgins JP, Thompson SG. Quantifying heterogeneity in a metaanalysis. Stat Med 2002;21:1539-58.

21. Egger M, Davey Smith G, Schneider M, et al. Bias in meta-analysis detected by a simple, graphical test. BMJ 1997;315:629-34.

22. Viera AJ, Garrett JM. Understanding interobserver agreement: the kappa statistic. Fam Med 2005;37:360-3.

23. Moher D, Liberati A, Tetzlaff J, et al. Preferred reporting items for systematic reviews and meta-analyses: the PRISMA statement. J Clin Epidemiol 2009;62:1006-12. 